TTP00-03

March 2000

\title{
Measuring $F_{L}\left(x, Q^{2}\right) / F_{2}\left(x, Q^{2}\right)$ from Azimuthal Asymmetries in Deep Inelastic Scattering
}

\author{
T. Gehrmann \\ Institut für Theoretische Teilchenphysik, Universität Karlsruhe, D-76128 Karlsruhe, \\ Germany
}

\begin{abstract}
We demonstrate that the angular distribution of hadrons produced in semiinclusive deep inelastic final states is related to the inclusive longitudinal structure function. This relation could provide a new method of accessing $F_{L}\left(x, Q^{2}\right)$ in deep inelastic scattering measurements.
\end{abstract}


The internal structure of the proton as probed in deep inelastic lepton-proton scattering can be parameterised in terms of two structure functions $F_{2}\left(x, Q^{2}\right)$ and $F_{L}\left(x, Q^{2}\right)$ :

$$
\frac{\mathrm{d} \sigma}{\mathrm{d} x \mathrm{~d} Q^{2}}=\frac{2 \pi \alpha^{2}}{x Q^{4}}\left[\left(1+(1-y)^{2}\right) F_{2}\left(x, Q^{2}\right)-y^{2} F_{L}\left(x, Q^{2}\right)\right] .
$$

$x$ and $Q^{2}$ are the usual deep inelastic scattering variables related to the momentum of the outgoing electron, and $y=Q^{2} /(x s)$, with $s$ being the lepton-proton centre-of-mass energy squared.

While measurements of $F_{2}\left(x, Q^{2}\right)$ have been made with high precision over a large kinematical range (see e.g. [1] for a review), experimental determinations on $F_{L}\left(x, Q^{2}\right)$ are still limited. Given that $F_{L}\left(x, Q^{2}\right)$, apart from encoding information on the internal structure of the proton, plays an important role in QED radiative corrections to processes in lepton-proton scattering, a precise determination of this structure function over a wide kinematical range would be very desirable.

The experimental measurement of $F_{L}\left(x, Q^{2}\right)$ in inclusive deep inelastic scattering is far from trivial, since the inclusive cross section (1) is dominated by the structure function $F_{2}\left(x, Q^{2}\right)$, with $F_{L}\left(x, Q^{2}\right)$ entering as a correction only. The contributions from $F_{2}\left(x, Q^{2}\right)$ and $F_{L}\left(x, Q^{2}\right)$ to the deep inelastic cross section can be disentangled only from their kinematical factors; a measurement of $F_{L}\left(x, Q^{2}\right)$ requires therefore the comparison of data at fixed $\left(x, Q^{2}\right)$ taken at different centre-of-mass energies. Such measurements have so far been carried out only at fixed target energies. A determination of $F_{L}\left(x, Q^{2}\right)$ at HERA energies has up to now been made only in an indirect manner [3] by assuming the contribution to the deep inelastic cross section from $F_{2}\left(x, Q^{2}\right)$ to be known from a next-to-leading order QCD fit.

In this note, we investigate an alternative possibility to determine $F_{L}\left(x, Q^{2}\right)$. We argue that the azimuthal distribution of hadrons in semi-inclusive deep inelastic scattering can be related to the longitudinal structure function by integrating out the final state hadron momentum in a appropriate way to recover inclusive expressions.

The cross section for semi-inclusive hadron production reads 4 , 5, 6]:

$$
\frac{\mathrm{d} \sigma}{\mathrm{d} x \mathrm{~d} Q^{2} \mathrm{~d} z \mathrm{~d} \phi}=\frac{\alpha^{2} \pi}{2 Q^{4} z}(A+B \cos \phi+C \cos 2 \phi) .
$$

$z$ is the longitudinal momentum transfer from virtual photon to the final state hadron, and $\phi$ is the angle between the outgoing lepton direction and outgoing hadron direction measured in the plane transverse to the virtual photon direction. The coefficients $A-C$ can be computed in the parton model, they correspond to convolutions of parton distributions $D_{a / N}$ in the target, hard subprocess coefficient functions $f^{b / a}$, and partonic fragmentation functions into the final state hadron $D_{h / b}$. While $A$ is of $\mathcal{O}(1)$ in perturbation theory, $B$ and $C$ are of $\mathcal{O}\left(\alpha_{s}\right)$. For neutral current deep inelastic scattering at HERA energies, one finds [7] that the coefficients of the angular dependent terms can be expected to amount up to $10 \%$ of the constant term, which is confirmed by a recent measurement 8 ] by the ZEUS collaboration. 
Momentum conservation in partonic fragmentation implies that the second moment of the fragmentation functions summed over all hadrons has to be unity for any parton $b$ :

$$
\sum_{h} \int_{0}^{1} \mathrm{~d} z z D_{h / b}\left(z, Q^{2}\right)=1
$$

This relation is preserved under perturbative evolution. Using this relation, it is possible to relate the second moment of any semi-inclusive observable to the corresponding inclusive observable. The use of this relation in deriving sum rules for semi-inclusive observables in deep inelastic scattering has first been suggested in [5].

Integrating over the angle $\phi$ and taking the second moment in $z$ of (2), one recovers the inclusive cross section (11):

$$
\sum_{h} \int_{0}^{1} \mathrm{~d} z \int_{0}^{2 \pi} \mathrm{d} \phi z \frac{\mathrm{d} \sigma}{\mathrm{d} x \mathrm{~d} Q^{2} \mathrm{~d} z \mathrm{~d} \phi}=\frac{2 \pi \alpha^{2}}{x Q^{4}}\left[\left(1+(1-y)^{2}\right) F_{2}\left(x, Q^{2}\right)-y^{2} F_{L}\left(x, Q^{2}\right)\right] .
$$

The coefficient of $\cos 2 \phi$ can be expressed by a semi-inclusive structure function [6]:

$$
C=(1-y) F_{6}\left(x, Q^{2}, z\right)
$$

with

$$
F_{6}\left(x, Q^{2}, z\right)=\frac{z}{\pi} \sum_{a, b} \int_{x}^{1} \frac{\mathrm{d} \xi}{\xi} \int_{z}^{1} \frac{\mathrm{d} \eta}{\eta} D_{h / b}\left(\eta, Q^{2}\right) D_{a / N}\left(\xi, Q^{2}\right) 4 e_{b / a}^{2} \frac{\alpha_{s}}{2 \pi} f_{6}^{b / a}\left(\frac{x}{\xi}, \frac{z}{\eta}\right),
$$

where

$$
\begin{aligned}
& f_{6}^{q / q}(\hat{x}, \hat{z})=f_{6}^{g / q}(\hat{x}, 1-\hat{z})=2 C_{F} \hat{x} \hat{z} \\
& f_{6}^{q / g}(\hat{x}, \hat{z})=4 T_{F} \hat{x}(1-\hat{x}) .
\end{aligned}
$$

In the above $q$ is representing a quark or antiquark.

Taking the second moment of $f_{6}^{b / a}(\hat{x}, \hat{z})$ with respect to $\hat{z}$ and summing over the final state parton, one recovers the longitudinal coefficient functions:

$$
\begin{aligned}
\sum_{b=q, g} \int_{0}^{1} \mathrm{~d} \hat{z} \hat{z} f_{6}^{b / q}(\hat{x}, \hat{z})=C_{F} \hat{x} & =\frac{1}{2} C_{L, q}^{(1)}(\hat{x}) \\
\sum_{b=q, \bar{q}} \int_{0}^{1} \mathrm{~d} \hat{z} \hat{z} f_{6}^{b / g}(\hat{x}, \hat{z})=4 T_{F} \hat{x}(1-\hat{x}) & =\frac{1}{2} C_{L, g}^{(1)}(\hat{x}) .
\end{aligned}
$$

Consequently, one finds that the ratio of the second moment of the coefficients of the $\cos 2 \phi$ term and the constant term in (2) yields:

$$
\begin{aligned}
A_{\cos 2 \phi}^{(2)}\left(x, Q^{2}\right) & \equiv \frac{\sum_{h} \int_{0}^{1} \mathrm{~d} z \int_{0}^{2 \pi} \mathrm{d} \phi z \cos 2 \phi \frac{\mathrm{d} \sigma}{\mathrm{d} x \mathrm{~d} Q^{2} \mathrm{~d} z \mathrm{~d} \phi}}{\sum_{h} \int_{0}^{1} \mathrm{~d} z \int_{0}^{2 \pi} \mathrm{d} \phi z \frac{\mathrm{d} \sigma}{\mathrm{d} x \mathrm{~d} Q^{2} \mathrm{~d} z \mathrm{~d} \phi}} \\
& =\frac{1}{2} \frac{(1-y) F_{L}\left(x, Q^{2}\right)}{\left(1+(1-y)^{2}\right) F_{2}\left(x, Q^{2}\right)-y^{2} F_{L}\left(x, Q^{2}\right)} .
\end{aligned}
$$


The ratio of the deep inelastic structure functions can therefore be expressed as

$$
\frac{F_{L}\left(x, Q^{2}\right)}{F_{2}\left(x, Q^{2}\right)}=2 \frac{1+(1-y)^{2}}{1-y+2 y^{2} A_{\cos 2 \phi}^{(2)}\left(x, Q^{2}\right)} A_{\cos 2 \phi}^{(2)}\left(x, Q^{2}\right)
$$

It should be noted that the sum over all hadrons $h$ in the above equations also includes neutral hadrons, whose momentum direction is difficult to track experimentally. Restricting the summation to charged hadrons only would introduce subprocess-dependent correction factors, relating to the different probabilities of quarks and gluons to fragment into charged hadrons, thus altering the relative magnitude of quark and gluon contributions in the above asymmetry. Furthermore, the summation includes only hadrons that participated in the hard scattering process, i.e. it excludes the proton remnant. Hadrons from the proton remnant will however emerge with low $z$, their contribution in the second moment can therefore be expected to be small.

In summary, we have demonstrated that the longitudinal structure function $F_{L}\left(x, Q^{2}\right)$ is related to the second moment of the angular $\cos 2 \phi$ distribution of hadrons produced in semi-inclusive deep inelastic scattering. The asymmetry defined in (9) provides a possibility of measuring the structure function ratio $F_{L}\left(x, Q^{2}\right) / F_{2}\left(x, Q^{2}\right)$ in a deep inelastic scattering experiment at fixed collision energy.

\section{Acknowledgement}

The author would like to thank Jim Crittenden and Nick Brook for pointing his attention to the issue discussed in this note and for numerous valuable discussions.

\section{References}

[1] T. Gehrmann, R.G. Roberts and M.R. Whalley, J. Phys. G25 Suppl. (1999) A1.

[2] L. Whitlow et al., Phys. Lett. B250 (1990) 193;

E140 Collaboration: S. Dasu et al., Phys. Rev. D49 (1994) 5641;

NMC Collaboration: M. Arneodo et al., Nucl. Phys. B487 (1997) 3;

E143 Collaboration: K. Abe et al., Phys. Lett. B452 (1999) 194.

[3] H1 Collaboration: C. Adloff et al., Phys. Lett. B393 (1997) 452.

[4] H. Georgi and H.D. Politzer, Phys. Rev. Lett. 40 (1978) 3.

[5] G. Köpp, R. Maciejko and P.M. Zerwas, Nucl. Phys. B144 (1978) 123;

A. Mendez, Nucl. Phys. B145 (1978) 199.

[6] K. Hagiwara, K. Hikasa and N. Kai, Phys. Rev. D27 (1983) 84.

[7] M. Ahmed and T. Gehrmann, Phys. Lett. B465 (1999) 297.

[8] ZEUS Collaboration: J. Breitweg et al., preprint DESY-00-040 (hep-ex/0003017). 